\title{
Molecular dynamics simulation of non-azeotropic refrigerants separation in auto-cascading refrigeration
}

\author{
Dayu Zheng, ${ }^{1, *}$, Shengnan Feng ${ }^{1}$, Liping $\mathrm{Gao}^{1}$ and Menglu $\mathrm{Li}^{1}$ \\ ${ }^{1}$ School of energy and architectural engineering,Harbin University of Commerce,china
}

\begin{abstract}
The core problem of auto-cascading refrigeration is the separation of non-azeotropic refrigerants. The separation effect of mixed refrigerant directly determines the refrigeration effect of auto-cascading refrigeration system and the value of COP. The microscopic molecular dynamics simulation method is applied to the refrigeration system, and the properties of the pure substance and the mixed substance are simulated from the microscopic aspects to study the law of molecular condensation cooling, and the separation rate is simulated to obtain a more optimized scheme.
\end{abstract}

\section{Introduction}

Auto-cascading refrigeration is one of the main ways to achieve a temperature range of $-60^{\circ} \mathrm{C} \sim-150^{\circ} \mathrm{C}$. It has very important applications in the fields of national defense military, modern industry, bio medicine and scientific research. Since the cascade system is in a dynamic stable state, the internal pressure is large, and the movement law of the material cannot be directly detected, the calculation of the system separation rate has been unable to be solved. In this paper, molecular dynamics simulation is used to simulate the process of refrigerant separation in the automatic cascade system to solve this problem.

\section{Auto-cascading refrigeration system}

As shown in Figure 2.1, the cycle diagram of the nonazeotropic mixed refrigerant auto-cascading refrigeration system used in this study. The specific process is described as follows: a certain proportion of nonazeotropic mixed working fluid is compressed into a high temperature and high pressure state in the compressor A, and then condensed in the condenser B. most R22 is condensed into liquid, while most medium boiling point working medium R23 and low boiling point working medium R14 are still gases. The mixture flows through a liquid-storage separator $E_{1}$, in which it is separated into gas-liquid phase. R22 components of liquid from the bottom of the separator, and the temperature in the separation tank $\mathrm{E}_{2}$ is controlled at about $-30^{\circ} \mathrm{C}$, most of the R23 gas in the tank is condensed into liquid, again by gravity make R23 liquid from the bottom of the tank $E_{2}$, the temperature control for tank $E_{2}$ is around $-70^{\circ} \mathrm{C} . \mathrm{R} 14$ components is the same process,finally, the evaporator temperature reached to $-90^{\circ} \mathrm{C}$, the evaporator of the air back through the plate heat exchanger in turn $F_{1}, F_{2}$ and $F_{3}$ arriving at gas-liquid separator into the compressor suction mouth, finishing a complete refrigeration cycle ${ }^{[1]}$.

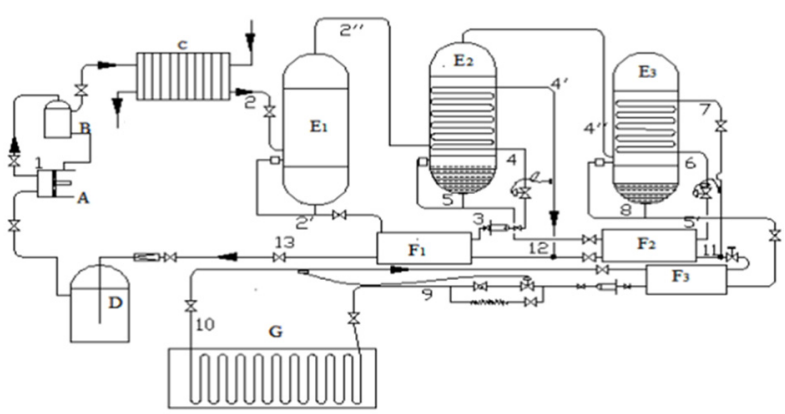

Figure 2.1 Flow chart of non-azeotropic mixed refrigerant autocascading refrigeration cycle

\section{Molecular dynamics simulation process}

\subsection{Potential function and molecular model}

The potential function used is the most widely used potential energy function Lennard-Jones (L-J) potential, which can be used to describe the interaction between molecules of inert gases and can also be used to describe some metals such as chromium, molybdenum. ${ }^{[2]}$ The equation of the potential function is:

$$
U_{i j}\left(r_{i j}\right)=A_{m}\left(\frac{d}{r_{i j}}\right)^{m}-B_{n}\left(\frac{d}{r_{i j}}\right)^{n}
$$

The molecular structure models R23 (molecular formula: $\mathrm{CHF}_{3}$ ) and R14 (molecular formula: $\mathrm{CF}_{4}$ ) were drawn by using the Chemsketch chemical software package according to the $\mathrm{C}-\mathrm{H}$ bond length of the methane $\mathrm{CH}_{4}$ molecule of $1.09 \AA$ and the $\mathrm{H}-\mathrm{C}-\mathrm{H}$ angle of $109.5^{\circ}$. We used the VMD visualization software and the balland-roll model to see the molecular structure of R23 and R14, as shown in Figure 3.1.

\footnotetext{
*Dayu Zheng: 18846033886@163.com
} 


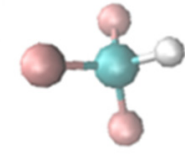

(a)R23
(b)R14

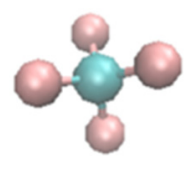

Figure 3.1: schematic diagram of molecular structure of R23 and R14

\subsection{Periodic boundary conditions and Berendsen's thermal bath method}

The Periodic Boundary Conditions reflect how boundary conditions can be used to replace selected parts (systems) from the surrounding environment ${ }^{[3]}$. It can be seen as the condition that if the surrounding environment is removed and the system is kept unchanged, it can also be regarded as part of the nature to promote the expression of the global nature. For this problem, the periodic boundary conditions can greatly improve the computational efficiency and increase the accuracy of the simulation calculations is fully applicable ${ }^{[4-5]}$.

The Berendsen thermal bath method is based on the principle that the system is in contact with an infinitely large temperature bath to maintain its temperature stability ${ }^{[6]}$. The equation for temperature change is:

$$
\Delta T=\frac{\delta t}{\tau}\left(T_{b a t h}-T(t)\right)
$$

calibration factor is:

$$
\lambda^{2}=1+\frac{\delta}{\tau}\left[\frac{T_{b a t h}}{T(t)}-1\right]
$$

This method allows the system temperature to float up and down at the desired temperature.

\subsection{Simulation process}

In order to test the accuracy of the model, we simulated the R23 and R14 pure substances separately. The simulation uses the GROMACS simulation software package to simulate refrigerants R23 and R14 under periodic boundary conditions. The pure material and mixture were then simulated under the control of a thermal bath at Berendsen with a cut-off radius of $11 \AA$ and a time step of $0.001 \mathrm{ps}$. The simulated control conditions are: pressure $3.5 \mathrm{MPa}$, temperature decreased from $281 \mathrm{~K}$ to $250 \mathrm{~K}$, and the equilibrium simulation time is $3 \mathrm{~ns} .1300$ R23 (or R14) molecules are randomly distributed in a cube box with a side length of $7 \mathrm{~nm}$, as shown in Figure 3.2. The figure shows the initial distribution of pure substance R23 particles in the box. Under the condition of constant pressure and temperature(NPT), the simulation reaches equilibrium. In order to calculate the refrigerant separation, an isothermal NVT(constant volume and temperature) simulation was performed for R23 and R14 mixtures to achieve equilibrium. Due to space constraints, we selected 1300 R23 and 137 R14 molecules to be placed in a mixed box according to the same ratio, and the simulation time was $3 \mathrm{~ns}$.

The initial distribution of molecules in the box of the size of refrigerant R23 at the beginning of MD simulation is shown in the figure below:

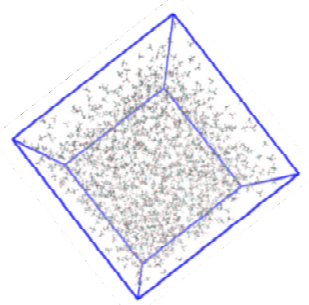

Figure 3.2 Distribution of pure material R23 initial position $(\mathrm{t}=0 \mathrm{~s})$

The size of the box when the mixed working fluids R23 and R14 start to simulate at the beginning of the MD, the initial distribution of molecules in the box as shown:

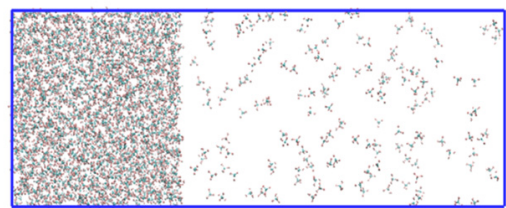

Figure 3.3 Initial position distribution of the mixture (R23-left, R14-right) ( $\mathrm{t}=0 \mathrm{~s})$

\subsubsection{Pure material simulation}

According to figure 3.4, we can see that the density value increases with time, and increases to a constant value of $156.86 \mathrm{~kg} / \mathrm{m}^{3}($ temperature $\quad \mathrm{T}=281 \mathrm{~K}) \quad$ and $1215.73 \mathrm{~kg} / \mathrm{m}^{3}$ (temperature $\left.\mathrm{T}=250 \mathrm{~K}\right)$. With the continuous extension of simulation time, this constant value almost remains unchanged, indicating that the simulation system has entered an equilibrium state. The simulation results are close to the data of NIST database $148.76 \mathrm{~kg} / \mathrm{m}^{3}$ (temperature $\left.\mathrm{T}=281 \mathrm{~K}\right)$ and $1197.6 \mathrm{~kg} / \mathrm{m}^{3}$ (temperature $\mathrm{T}=250 \mathrm{~K}$ ), indicating that the molecular and potential functions of R23 simulated by MD are consistent with the actual substance and can meet the calculation needs.

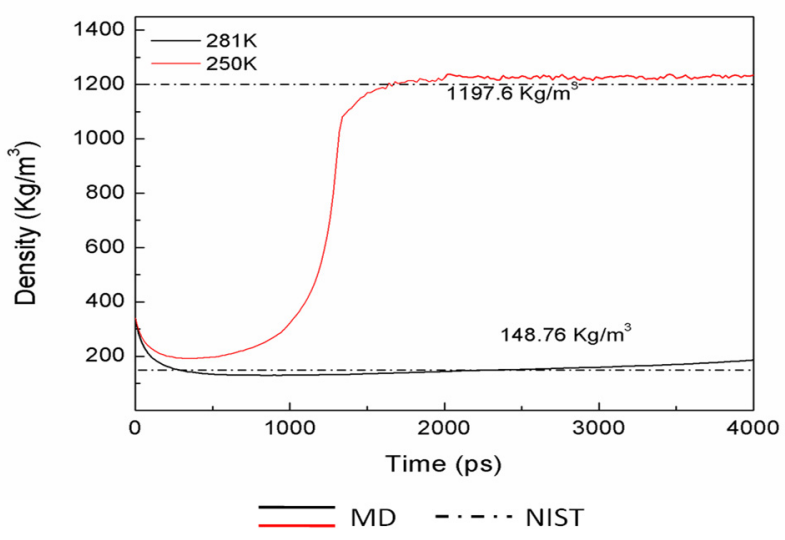

Figure 3.4 R23 density versus time graph 
The solid line in the figure represents the MD simulation results, and the dotted line represents the NIST database data. From the figure, we can find that the simulated data is slightly different from the standard data, but it can be used to describe the properties of materials. It can be used in MD mixture simulation.

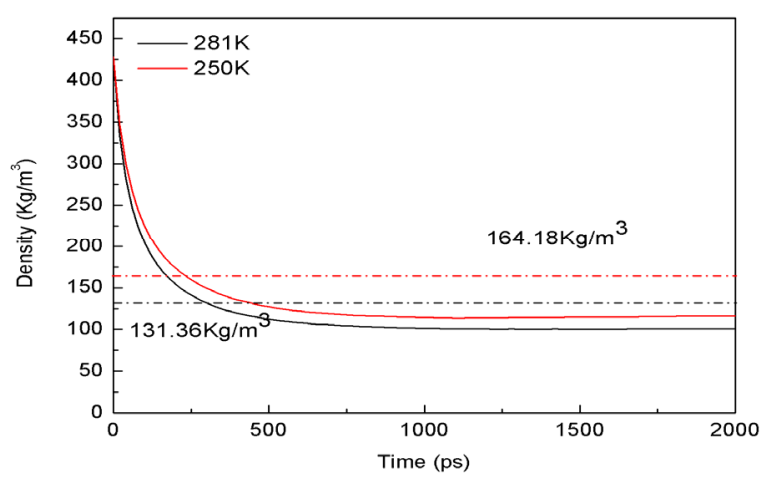

Figure 3.5 R14 density versus time graph

Figure 3.5 describes the relationship between the density and time of refrigerant R14 at a constant pressure of $281 \mathrm{~K}$ and $250 \mathrm{~K}$.

\subsubsection{Simulation of mixed substances}

According to the equal mass ratio of the two refrigerants, 104 R23 particles and 83 R14 particles were selected to mix to make the simulation run 3 ns under NPT conditions. The density changes with time at temperatures of $281 \mathrm{~K}$ and $250 \mathrm{~K}$ were obtained as shown in figure 3.6.

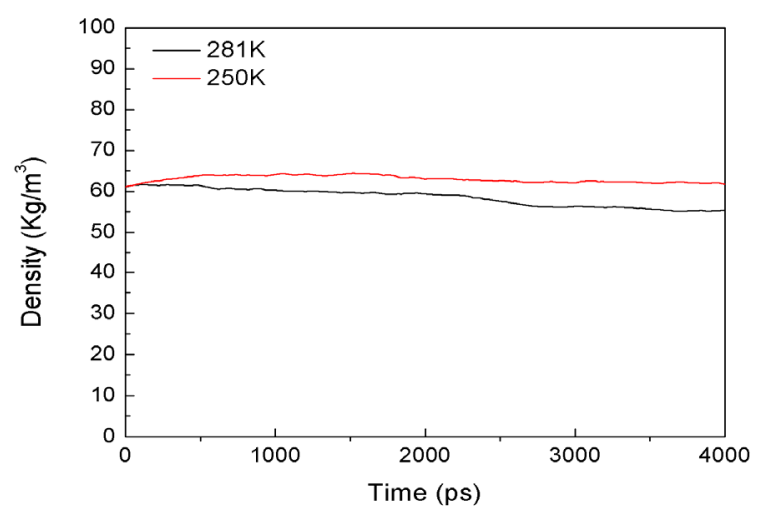

Figure. 3.6 Mixture density versus time

According to this figure, the density increases with time and remains almost unchanged, indicating that the simulation system has entered an equilibrium state. Figure 3.7 shows the particle position distribution diagram when the simulated time $\mathrm{t}=3 \mathrm{~ns}$, and the temperature (a) is $281 \mathrm{~K}$ and the temperature (b) is $250 \mathrm{~K}$. In order to distinguish the two particle distributions, we use red and blue to represent R23 and R14, respectively. By comparing the figures (a) and (b), we can find that the mixture volume decreases as the temperature decreases, which indicates that the distance between molecules decreases. But since the system is at rest, it is difficult to accurately judge the separation of the two substances. (a)

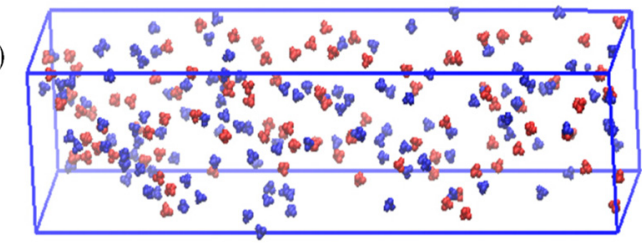

2

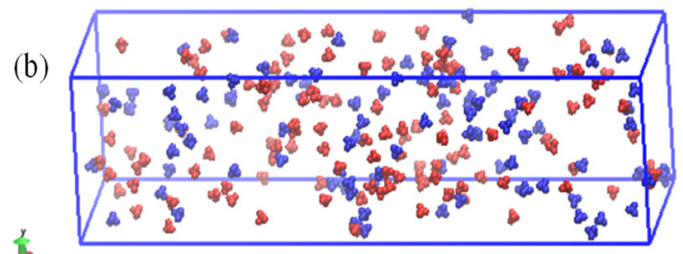

Figure 3.7 (a) $281 \mathrm{~K}$ particle position distribution (b) $250 \mathrm{~K}$ particle position distribution

According to the equal volume ratio of the two refrigerants, 1300 R23 particles and 137 R14 particles are mixed. Under the control of NVT conditions, the simulation time is $3 \mathrm{~ns}$, and the vapor-liquid separation of the mixture is studied according to the diffusion principle. Figure 3.8 depicts the positional distribution of particles at (a) $\mathrm{t}=0 \mathrm{~ns}$ and (b) $\mathrm{t}=3 \mathrm{~ns}$ at a temperature of $250 \mathrm{~K}$. Observing this figure, it is found that the interface between the two substances is fused because the diffusion of the two substances occurs at rest. A part of the gas diffuses into the liquid, and a part of the liquid also diffuses into the gas, thus forming a vapor-liquid mixing interface. According to the diffusion principle, the separation rate of the mixture can be solved.
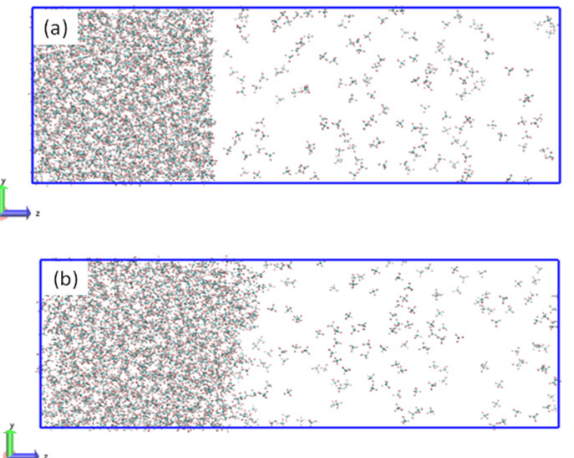

Figure. 3.8 Particle position distribution diagram of the system $\begin{array}{ll}\text { (a) Time } \mathrm{t}=0 \mathrm{~ns} & \text { (b) Time } \mathrm{t}=3 \mathrm{~ns}\end{array}$

Figure 3.9 shows the density distribution in the z-axis direction. With the increase of the z-axis, the density of the system increases to a certain density value of 1082.46 $\mathrm{kg} / \mathrm{m}^{3}$, and then the density value begins to rapidly decline to a constant value of $63.45 \mathrm{~kg} / \mathrm{m}^{3}$. The critical values of the simulated interface are $978.63 \mathrm{~kg} / \mathrm{m}^{3}$ and $71.42 \mathrm{~kg} / \mathrm{m}^{3}$ respectively. The dark blue dotted line in the figure indicates the location of the interface. 


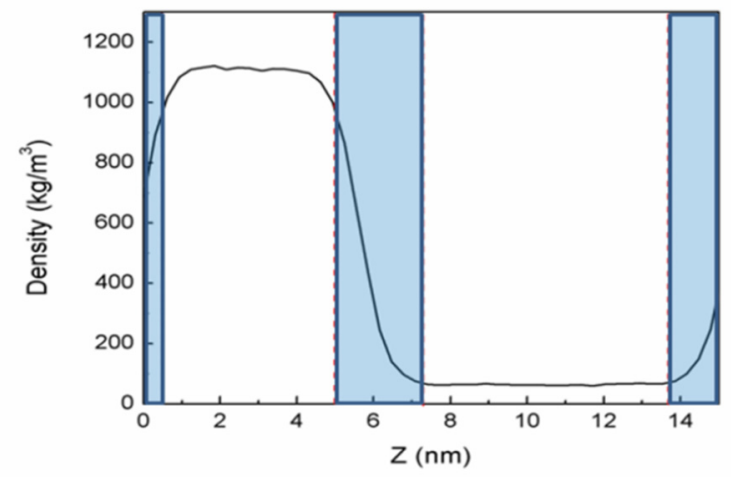

Figure 3.9 Relationship between density and $\mathrm{z}$ value

We use the distribution of particles in the interface to calculate the separation of matter. The separation rate of a substance in the mixture is calculated as follows:

$$
\eta=\frac{n \cdot M}{\rho \cdot N_{A} \cdot v} \times 100 \%
$$

According to the mass density distribution of the refrigerants R23 and R14, the separation rates of R23 and R14 were calculated to be $21.36 \%$ and $32.86 \%$, respectively, using the separation ratio (3-4). Empirically, the separation rate is $10 \%-20 \%$ for better separation. In order to find the optimum separation rate, the cooling amount that provides condensation and the ratio of R23/R14 were changed and simulated again.

When the condensing temperature is changed, the temperature of R23/R14 changes when R22 provides cooling capacity, and the separation rate is $22.73 \%$ and $35.42 \%$ when the temperature is $281 \mathrm{~K}-250 \mathrm{~K}$. The ratio of different R23/R14 was used for simulation, and the ratio separation rate of $1: 1$ was $35.42 \%$, which was the maximum.

\section{Conclusion}

For the ternary non-azeotropic mixture in the autocascading refrigeration system, when the cooling capacity of the condensing is changed and the ratio of the refrigerant is changed, the separation effect is changed. By analyzing the molecular dynamics simulation results, when R22 provides cooling capacity, the separation rates of R23/R14 changes accordingly, and the separation rate of temperature from $281 \mathrm{~K}$ to $250 \mathrm{~K}$ is $22.73 \%$ and $35.42 \%$. The simulation was carried out with different ratios of $\mathrm{R} 23 / \mathrm{R} 14$, and the ratio of separation ratio obtained at 1:1 was $35.42 \%$. The more cooling is provided and the more R23 is condensed out. Similarly, R23 condenses into the lower stage for final cooling. The R14 refrigerant is larger and the system COP is also increased.

\section{References}

1. Dayu Zheng, Yiming Zhang, Xin Chen, et al. Experimental study of R22/R23/R14 three-stage automatic cascade refrigeration system $[\mathrm{J}]$. Journal of
Harbin University of Commerce: Natural Science Edition, 2012, 28(6): 716- 718.

2. Kai Jiang, Yunfei Chen, Ping Zhuang, et al. Molecular Dynamics Simulation of Nanoscale Boundary Slip[J]. Journal of Tribology, 2005, 25(3): 238-242.

3. Hailan Shen, Jingsong Zhao . Overview of Molecular Dynamics Simulation Methods[J]. Equipment Manufacturing Technology, 2007 (10): 29-30.

4. Ping Yang, Yimin Sun. Molecular Dynamics Simulation Method and Its Application[J]. Journal of Anhui Normal University: Natural Science Edition, 2009, 32(1): 51-54.

5. Chunyan Li, Hua Liu, Botao Liu . Fundamental Principles and Research Progress of Molecular Dynamics Simulation[J]. Guangzhou Chemical, 2011, 39(4): 11-13.

6. Xiaoping Zhou, Zhuangzhuang Tian, Xiaowei Xu . Overview of Molecular Dynamics Simulation Methods[J]. Silicon Valley, 2012 (23): 40-41. 